\title{
Molecular beam epitaxial growth of semiconductor heterostructures for THz electronics
}

\author{
Victor M. Ustinov ${ }^{1,2}$ \\ ${ }^{1}$ Ioffe institute, St. Petersburg, Russia, vmust@beam.ioffe.ru \\ ${ }^{2}$ SHM R\&E Center, RAS, St. Petersburg, Russia
}

In recent two decades semiconductor heterostructures emerged as a very promising basis for the development of various types of devices in a microwave frequency range. In the present work we discuss molecular beam epitaxial (MBE) growth of wide miniband superlattice (SL), heterostructure barrier varactor (HBV) and high electron mobility transistor (HEMT) structures, their characterization and application of these heterostructures for generators, detectors and frequency multipliers operating in the sub- $\mathrm{THz}$ and $\mathrm{THz}$ frequency range.

\section{Superlattices.}

The superlattice structures were grown by solid source molecular beam epitaxy using a Riber 32 machine. GaAs based structures were grown on GaAs substrates, consisted of several tens of periods of GaAs wells (3 - $4 \mathrm{~nm}$ thick) and AlAs barriers ( about $1 \mathrm{~nm}$ thick) doped with silicon $\left(10^{17}-10^{18} \mathrm{~cm}^{-3}\right)$. The superlattice was embedded between graded layers of gradual composition and doping level to avoid abrupt heterojunctions. A $\mathrm{n}^{+} \mathrm{GaAs}$ or GaAs/InGaAs cap layer was provided for the formation of ohmic contacts. InP based SL structures were grown lattice matched to InP substrates and consisted of several tens of periods of $\mathrm{In}_{0.53} \mathrm{Ga}_{0.47} \mathrm{As}$ wells (about $4 \mathrm{~nm}$ thick) and $\mathrm{In}_{0.52} \mathrm{Ga}_{0.48} \mathrm{As}$ barriers (about $1.2 \mathrm{~nm}$ thick) doped with silicon $\left(10^{17}-10^{18} \mathrm{~cm}^{-3}\right)$. The superlattice was embedded between graded layers of gradual composition and doping level to avoid abrupt heterojunctions. InGaAs cap layer was provided for the formation of ohmic contacts. Mesa diodes (Fig. 1) with area of about 60 and $10000 \mu \mathrm{m}^{2}$ were formed using $\mathrm{Au}-\mathrm{Ge}-$ Ni composition [1].

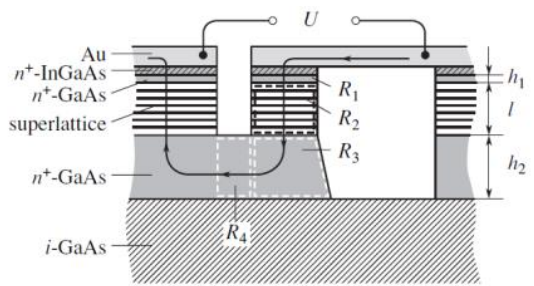

Fig. 1. Cross section of the GaAs based diode structure

Changing the widths of the well and barrier layers the lowest SL miniband width could be varied from about 20 up to $160 \mathrm{meV}$. These doped wide miniband superlattices exhibit negative differential conductance (NDC), Fig. 2, which is a consequence of Bragg reflection of miniband electrons accelerated by an electric field.

The negative differential mobility of a superlattice can give rise to travelling dipole domains and a self-sustained current oscillation at the transit fre- quency, which is the ratio of the domain velocity and the superlattice length. The fundamental oscillation frequency increased with the increase in the miniband width due to the increase of peak drift velocity [2]. The oscillation frequency can be controlled by changing the bias voltage applied to the diode. In comparison to a GaAs/AlAs superlattice, an InGaAs/InAlAs superlattice with the same quantum well and barrier thickness has the advantage of a larger miniband and, therefore, of a higher drift velocity due to the lower effective electron masses of the constituent materials and a lower barrier height. The highest fundamental frequency of an InGaAs/InAlAs superlattice oscillator is shown on Fig. 3. The power was $\sim 80 \mathrm{~mW}$ corresponding to a conversion efficiency from DC to highfrequency power of $0.6 \%$ [3].

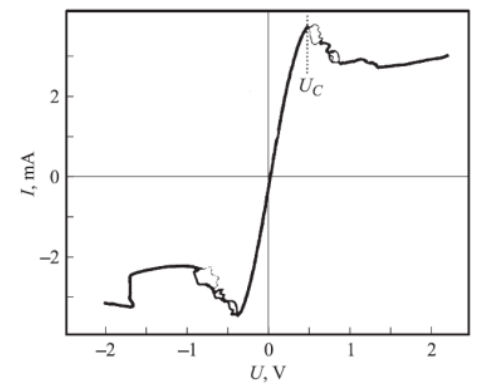

Fig. 2. Current-voltage characteristic of SL diode

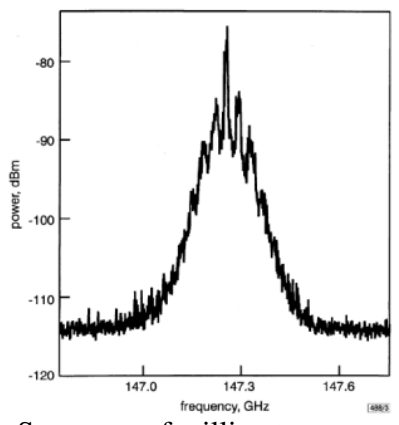

Fig. 3. Spectrum of millimetre wave emission from InGaAs/InAlAs superlattice device.

Strongly nonlinear U-V characteristic in the NDC region allows the use of the doped wide miniband superlattice device as a frequency multiplier in the microwave emission range, Fig. 4. The input frequency $\mathrm{F}=150 \mathrm{GHz}$. Maximum harmonic number is $\mathrm{n}=$ 54 , maximum harmonic frequency is $8.1 \mathrm{THz}$. Most part of radiation power of the frequency multiplier is concentrated in first 10 harmonics between 0.4 and $1.5 \mathrm{THz}[4]$.

It was found that $\mathrm{THz}$ radiation affects Bloch oscillating miniband electrons. The direct current through a superlattice is reduced by a $\mathrm{THz}$ field and 
resonant structures in the current-voltage characteristic occur when the Bloch frequency is in resonance

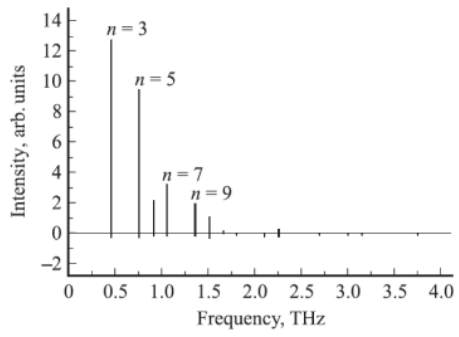

Fig. 4. GaAs/AlAs SL frequency multiplier harmonics intensity in the $0.4-4.1 \mathrm{THz}$ range.

with the $\mathrm{THz}$ field or with its harmonics. In comparison to a GaAs/AlAs superlattice detector, the InGaAs/InAlAs superlattice detector has a responsivity, which is higher by an order of magnitude. The higher responsivity is caused by a higher current density due to a larger miniband of the InGaAs/InAlAs superlattice [5].

\section{Heterostructure barrier varactors.}

Heterostructure barrier varactor (HBV), Fig. 5, frequency multipliers demonstrate very promising results for applications in sub- $\mathrm{THz}$ and $\mathrm{THz}$ range. The best results for HBV multipliers have been achieved using MBE-grown heterostructures on InP substrates with several $\mathrm{In}_{0.52} \mathrm{Al}_{0.48} \mathrm{As} / \mathrm{AlAs} / \mathrm{In}_{0.52} \mathrm{Al}_{0.48} \mathrm{As}$ barrier layers in the $\mathrm{In}_{0.53} \mathrm{Ga}_{0.47} \mathrm{As}$ matrix. We have found that leakage current strongly decreases with the increase in the AlAs insertion thickness due to the reduction in the electron tunneling probability. Strain compensation InGaAs layers also decrease the leakage current at the same AlAs thickness. This may be attributed to the positive effect of the decrease in the overall amount of strain in the structure and better structure quality. Frequency tripler was fabricated using 6 mesa, 18 barrier HBV diode, with a cross section area of $7 \times 150 \mu \mathrm{m}^{2}$. Under CW operation at $94 \mathrm{GHz}$ output frequency the maximal output power exceeds $100 \mathrm{~mW}$ at the conversion efficiency of about $14 \%$. The estimated value of HBV cut-off frequency exceeds $1.2 \mathrm{THz}$ [6].

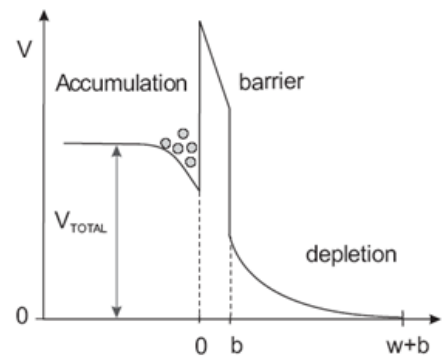

Fig. 5. HVB conduction band diagram at applied bias voltage.

\section{HEMTs.}

Nonlinear properties of the plasmonic excitations in a two-dimensional electron channel of HEMT can be used for detection of terahertz $(\mathrm{THz})$ radiation. The plasmonic FET THz detectors are promising because they can operate in a broad $\mathrm{THz}$ frequency range at ambient temperature and exhibit a good balance between the responsivity and noise figure of merit. Along with the plasmonic nonlinearity, the asymmetry of the boundary conditions at the source and drain ends of the HEMT electron channel is mandatory for enabling a non-zero detection response. We fabricated a tight concatenation of four HEMT s connected in series on a single chip. It is shown that such HEMT chain exhibits strong $\mathrm{THz}$ photovoltaic response due to asymmetric form of the T-gate in each HEMT in the chain. We obtained the voltage responsivity above $1 \mathrm{kV} / \mathrm{W}$ in the unbiased mode of the detector operation [7].

\section{References}

1.D. G. Pavel'ev, N. V. Demarina, Yu. I. Koshurinov, A. P. Vasil'ev, E. S. Semenova, A. E. Zhukov, V. M. Ustinov Characteristics of planar diodes based on heavily doped GaAs/AlAs Superlattices in the terahertz frequency region // Semiconductors. 2004. V. 38, No. 9. P. 1105-1110.

2. E. Schomburg, T. Blomeier, K. Hofbeck, J. Grenzer, S. Brandl, I. Lingott, A. A. Ignatov, K. F. Renk, D. G. Pavel'ev, Yu. Koschurinov, B. Ya. Melzer, V. M. Ustinov, S. V. Ivanov, A. Zhukov, P. S. Kop'ev Current oscillation in superlattices with different miniband widths // PHYS. REV. B. 1998. V. 58, No. 7. P. $4035-4038$.

3. E. Schomburg, R. Scheuerer, S. Brandl, K.F. Renk, D.G. Pavel'ev, Yu. Koschurinov, V. Ustinov, A. Zhukov, A. Kovsh, P.S. Kop'ev InGaAs/InAlAs superlattice oscillator at $147 \mathrm{GHz} / /$ Electron. Lett. 1999. V. 35, No. 17. Online No: 19990973

4. D. G. Paveliev, Y. I. Koshurinov, A. S. Ivanov, A. N. Panin, V. L. Vax, V. I. Gavrilenko, A. V. Antonov, V. M. Ustinov, A. E. Zhukov Experimental study of frequency multipliers based on a GaAs/AlAs semiconductor superlattices in the terahertz frequency range // Semiconductors. 2012. V. 46, No. 1 P. 121-125.

5. E. Schomburg, F. Klappenberger, M. Kratschmer, A. Vollnhals, R. Scheuerer, K.F. Renk, V. Ustinov, A. Zhukov, A. Kovsh InGaAs/InAlAs superlattice detector for $\mathrm{THz}$ radiation // Physica E. 2002. V. 13, P. $912-915$

6. N. A. Maleev, V. A. Belyakov, A. P. Vasil'ev, M. A. Bobrov, S. A. Blokhin, M. M. Kulagina, A. G. Kuzmenkov, V. N. Nevedomskii, Yu. A. Guseva, S. N. Maleev, I. V. Ladenkov, E. L. Fefelova, A. G. Fefelov, V. M. Ustinov Molecular-Beam Epitaxy of InGaAs/InAlAs/AlAs Structures for Heterobarrier Varactors // Semiconductors 2017. V. 51, No. 11. P. $1431-1434$.

7. V. V. Popov, D. M. Yermolaev, K. V. Maremyanin, V. E. Zemlyakov, N. A. Maleev, V. I. Gavrilenko, V. A. Bespalov, V. I. Yegorkin, V. M. Ustinov, S. Yu. Shapoval Detection of terahertz radiation by tightly concatenated InGaAs fieldeffect transistors integrated on a single chip // Appl. Phys. Lett. 2014. V.104, 163508 